\title{
Une analyse modale de type stochastique sous-espace avec prévision de l'erreur statistique sur les amortissements
}

\author{
Camille Gontier ${ }^{\mathrm{a}}$ et Roger Clara-Serra \\ Laboratoire de Mécanique et Rhéologie, Université de Tours, École d'ingénieurs du Val de Loire, Rue de la Chocolaterie, \\ 41034 Blois Cedex, France
}

Reçu le 2 octobre 2002, accepté le 6 janvier 2004

\begin{abstract}
Résumé - De plus en plus fréquemment, l'analyse modale d'une structure en laboratoire est complétée, voire remplacée, par une analyse modale de la structure «en service ». Les sollicitations du système sont alors presque toujours inconnues. On pratique alors un mode d'analyse dit «purement stochastique ». Les caractéristiques modales identifiées sont alors également de nature stochastique. Les taux d'amortissement, qui déjà, dans des conditions déterministes, sont souvent obtenus avec une faible précision, se trouvent alors fortement sujets à caution. Il est donc souhaitable d'assortir ces résultats d'une fourchette de validité statistique, et cela sans avoir recours à une coûteuse campagne statistique de résultats. Certains auteurs (Viberg, Jansson, ....) ont montré que la procédure d'identification elle-même est capable de fournir cette fourchette. Leur approche est ici mise en application dans le cas de l'identification des amortissements et est prolongée dans le cadre de l'approche stochastique de Van Overshee-De Moor. Deux validations en simulation, et une validation expérimentale sont présentées.
\end{abstract}

Mots clés : Analyse modale / identification / méthode sous-espace / stochastique / amortissement

Abstract - Stochastic subspace modal analysis with prediction of the damping ratio dispersions. Classical "on bench" modal analysis of structures is increasingly completed, or even replaced, by their "on line" modal analysis. In this case the system excitations are generally unknown and the useful identification procedure is declared as "pure stochastic". The identified modal characteristics are therefore also of a stochastic nature. Identified damping ratios, which in determistic conditions are often obtained with bad accuracy, may be somewhat unreliable. It is thus important to complete these results with an estimation of the dispersion of these values, without requiring a costly statistical campaign. A few authors (Viberg, Jansson, ... ) have shown that the identification procedure itself is able to provide an estimate of the dispersion. Their approach is applied here in the case of the damping ratio identification and is extended to Van Overshee-De Moor's approach. Two validations in simulation, and an experimental one are presented and discussed.

Key words: Modal analysis / identification / subspace method / stochastic / damping ratio

\section{Introduction}

Parmi les méthodes d'analyse modale disponibles à ce jour, on trouve, en alternative aux méthodes fréquentielles traditionnelles [1], un certain nombre de méthodes temporelles dites «paramétriques » $[2-5]$, connues sous les étiquettes «AR, ARX, ARMA, ... *, et d'autres méthodes dites «d'équation d'état ». Ces dernières se présentent encore sous deux aspects différents : d'une part les méthodes dites « de réalisation», telles la méthode ERA [6], d'autre part les méthodes dites

\footnotetext{
a Auteur correspondant : gontier@univ-tours.fr
}

« de sous-espace », connues sous les étiquettes 4SID [7], N4SID [8,9], MOESP [10], ... Certaines méthodes plus anciennes telles que CVA (Larimore), Composantes Principales, peuvent être rattachées à l'une ou l'autre ces familles précédentes.

Parmi ces méthodes, les méthodes de sous-espace offrent un attrait particulier, en ce sens que contrairement à la plupart des autres, elles ne supposent aucune manipulation préalable des données avant analyse (transformée de Fourier, reconstruction de réponses impulsionnelles...). Parmi elles, les méthodes de sousespace dites «stochastiques » occupent une place particulière [11]. Dans ce contexte, aucune information sur 
les excitations du système n'est disponible. Ces excitations ont en général un caractère diffus. C'est le cas par exemple des analyses dites « en ligne » pour les engins de transport : trains, avions... les excitations sont exercées en de nombreux points de la structure par suite d'interactions complexes avec l'environnement. Il existe pourtant des méthodes permettant l'identification modale dans ces situations. L'excitation étant de nature stochastique, les résultats sont, naturellement, de nature stochastique, de sorte qu'une identification unique ne fournit en fin de compte que des informations d'assez faible valeur. En particulier aucune indication n'est alors disponible quant à la validité de ces informations. Pourtant, certains auteurs ont compris que l'analyse elle-même était capable de fournir en même temps les paramètres identifiés et une information sur la validité de ces estimations.

Le problème de la validité statistique des informations est particulièrement critique en ce qui concerne l'identification des petits amortissements. L'expérience montre que cette information est obtenue avec une très grande dispersion relative. Il est pourtant des domaines où cette information est cruciale, ainsi celui de l'aéronautique, pour lequel l'approche des amortissements nuls est une situation redoutée connue sous le nom de «flottement ».

L'objet de cet article est précisément de proposer un outil permettant d'anticiper la validité d'une estimation statistique (fourchette), à partir d'une analyse unique. Après un rappel des principes de l'analyse stochastique, la théorie permettant cette validation statistique sera développée, puis mise en application sur des exemples en simulation et sur un cas expérimental.

\section{Procédure générique}

Dans ce paragraphe sera introduite une notation générique sous-forme de 5 procédures (P1)-(P5). Ces notations seront utilisées par la suite tout au long de l'article.

Pour un signal discrétisé quelconque $s(t)$; deux index $\alpha, k$, une longueur de signal $N$ étant donnés, un vecteur étendu $s_{k, \alpha}$ et une matrice de Hankel du signal $S^{\alpha}$ sont définis suivant les procédures suivantes :

$$
\begin{aligned}
s_{k, a}= & {\left[\begin{array}{c}
s(k) \\
s(k+1) \\
\ldots \\
s(k+\alpha-1)
\end{array}\right] ; } \\
S^{\alpha}= & {\left[\begin{array}{cccc}
s(1) & s(2) & \ldots & s(N) \\
s(2) & s(3) & \ldots & s(N+1) \\
\ldots & \ldots & \ldots & \ldots \\
s(\alpha) & s(\alpha+1) & \ldots & s(N+\alpha-1)
\end{array}\right] }
\end{aligned}
$$

Deux index $\alpha$ et $\beta$ étant donnés, autour d'un instant $k$, un vecteur « signal du passé » et un vecteur «signal du futur $»$ sont définis à partir de la procédure $(\mathrm{P} 1)$ de la manière suivante :

$$
s^{p}=s_{k, \alpha} \quad s^{f}=s_{k+\alpha, \beta}
$$

Enfin des matrices de Hankel « du passé » et « du futur » sont définies à partir de la procédure (P2)

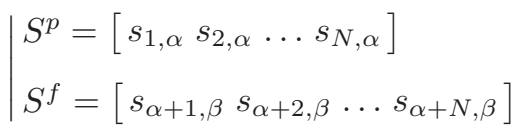

Une notation simplifiée sera utilisée pour des matrices ne contenant qu'une bloc-ligne. On notera

$$
S_{i}=[s(i) s(i+1) \ldots s(i+N-1)]
$$

\section{Les méthodes de sous-espace}

\subsection{Classification}

Les méthodes de sous-espace sont généralement réparties en 3 grandes variantes : les méthodes de type « déterministe » [12], les méthodes de type «stochastique » [12], et les méthodes de type « déterministestochastique »[13]. Les méthodes « déterministes », mais aussi les méthodes de type « déterministe-stochastique » considèrent les bruits de process et de mesure comme des perturbations, négligeables dans le premier cas, prises en compte directement dans le second cas. La situation typique est celle d'une analyse de laboratoire, dans des conditions expérimentales relativement bonnes.

Les méthodes de type «purement stochastique » sont en fait assez différentes des précédentes par le contexte applicatif. On les dit souvent «sous excitation inconnue ». Le contexte applicatif est typiquement le suivant : la structure est analysée « en-service », ainsi lorsque le wagon roule, ou lorsque l'avion vole ... Il est clair que dans ces cas les signaux d'excitation sont définitivement inconnus, généralement diffus sur toute la structure du système : interactions aérodynamiques sur les coques, réactions des rails et du ballast sur les roues, ... On pose généralement l'équation d'état du système sous la forme :

$$
\mid \begin{aligned}
& \dot{x}=\underline{A} x+w \\
& y=\underline{C} x+v
\end{aligned}
$$

où l'on considère que les signaux $w$ et $v$ sont des bruits blancs.

Il y a cependant deux remarques importantes à faire. D'abord il y a lieu de souligner que le système analysé n'est plus une structure bien définie, mais généralement un ensemble large comportant par exemple un véhicule, l'air ambiant (excitation aérodynamique) et le support (route, rail, ballast, ... ). De tout cet ensemble, en l'absence d'informations intermédiaires, il y a évidemment peu d'espoir d'extraire le comportement du sous-système «véhicule». Ce fait peut être de peu d'importance dans la mesure où l'intérêt de l'objet est précisément de rouler ou de voler. L'inconvénient par contre est que le résultat peut être difficile à extrapoler lorsque la même structure serait placée dans des conditions ambiantes différentes : vitesse, qualité des voies... 
Une deuxième remarque concerne le choix de la représentation de l'excitation par un «bruit blanc». Il est clair qu'en général, l'excitation supportée par la structure n'a pas cette propriété. De fait, le « bruit d'excitation », inconnu, a lui-même « une dynamique », que l'on ne saurait séparer de la dynamique de l'ensemble. Pour le système, cela se traduit par une extension de la dimension de l'espace des états. Le concept de bruit blanc est donc en fin de compte un concept «formel », destiné à représenter la part «non-expliquable»du comportement.

On voit donc que le contexte « purement stochastique » se présente sous un aspect très différent des deux contextes précédents. Le terme de bruit stochastique y est en fait la seule sollicitation du système, et joue alors un rôle central. Les seules informations recueillies sont les « sorties » du système. La section suivante rappellera les moyens d'en extraire les informations modales.

\section{L'analyse de sous-espace stochastique}

\section{1 Équations d'état}

Sous l'hypothèse de petits déplacements, l'équation d'état d'une structure s'exprime sous la forme : $M \ddot{x}+$ $C \dot{x}+K x=f$ où $M, C, K$ sont respectivement les matrices de masse, amortissement, et raideur de la structure, $x$ et $f$ les vecteurs des déplacements et forces extérieures.

Les étapes de mise sous forme d'équation d'état puis discrétisation sont classiques [13], et ne seront pas rappelées ici. La dynamique du système se présente alors sous la forme :

$$
\mid \begin{aligned}
& x(k+1)=A x(k)+B u(k) \\
& y(k)=C x(k)+D u(k)
\end{aligned}
$$

Dans cette équation $f(k)$ et $y(k)$ représentent les vecteurs excitations et réponses observées à l'instant $k, A, B, C$, $D$ les matrices définissant la dynamique du système. De manière classique, les valeurs propres et vecteurs propres de la matrice $A$ fournissent les pulsations modales, amortissements modaux et déformées modales de la structure.

Dans le contexte de l'analyse stochastique, les excitations $u(k)$ sont inconnues. De la discussion de la section 3.1 il résulte que la dynamique du système peut être posée sous la forme

$$
\mid \begin{aligned}
& x(k+1)=A x(k)+w(k) \\
& y(k)=C x(k)+v(k)
\end{aligned}
$$

où $w(k)$ et $v(k)$ représentent des bruits blancs de moyenne nulle, que nous appellerons « bruits de système ».

\subsection{Principe des méthodes de sous-espace}

Considérons le système défini par l'équation d'état (1). Cette équation peut être réécrite sous une forme fondamentale, qui est à la base de toutes les méthodes de sousespace. Pour un choix de deux indices $k, \alpha$ arbitraires, on construit un vecteur de « réponse » $y_{k, \alpha}$ défini par la procédure (P1) ci-dessus. On peut aisément montrer récursivement l'équation suivante :

$$
y_{k, \alpha}=\Gamma_{\alpha} x(k)+n_{k, \alpha}
$$

où l'on a défini un vecteur de bruit unique $n_{k, \alpha}$ :

$$
n_{k, \alpha}=\Psi_{\alpha} w_{k, \alpha}+v_{k, \alpha}
$$

et deux matrices caractéristiques du système :

$$
\Gamma_{\alpha}=\left[\begin{array}{c}
C \\
C A \\
\cdots \\
C A^{\alpha-1}
\end{array}\right]
$$

$$
\Psi_{\alpha}=\left[\begin{array}{ccccc}
0 & 0 & 0 & \ldots & 0 \\
C & 0 & 0 & \ldots & 0 \\
C A & C & 0 & \ldots & 0 \\
\cdots & \cdots & \cdots & \ldots & \ldots \\
C A^{\alpha-2} & C A^{\alpha-3} & C A^{\alpha-4} & \ldots & 0
\end{array}\right]
$$

La matrice $\Gamma_{\alpha}$ est dite « matrice d'observabilité étendue » et joue un rôle fondamental dans l'analyse. En raison du terme $n_{k, \alpha}$, l'équation (2) ne permet pas d'extraire directement cette matrice. On a alors recours à deux dispositions : i) élimination du bruit. On utilise en général la méthode dite « de la variable instrumentale », ii) réduction de la dimension d'espace d'état. L'outil généralement utilisé est la décomposition en valeurs singulières (SVD).

Le principe de la variable instrumentale consiste à corréler le signal de réponse $y$ avec un signal qui lui soit fortement corrélé, mais qui ne soit pas corrélé avec les bruits du système. En général, ce signal est obtenu en décalant le signal de réponse d'un délai temporel suffisant. C'est le principe de la méthode N4SID.

De manière pratique, le signal de réponse est partagé autour d'un instant $k$ entre un vecteur $y^{\mathrm{p}}$ et un vecteur $y^{\mathrm{f}}$ respectivement appelés «observation du passé » et « observation du futur », et définis par la procédure générique $(\mathrm{P} 3)$. Un vecteur « bruit du futur $n^{\mathrm{f}}$ étant défini de la même manière, l'équation fondamentale (2) s'écrit, dans le domaine « du futur »:

$$
y^{\mathrm{f}}=\Gamma_{\beta} x(k+\alpha)+n^{\mathrm{f}}
$$

La variable instrumentale choisie est celle « du passé » soit $y^{\mathrm{p}}$, non corrélée avec le bruit $n^{\mathrm{f}}$ de sorte que l'on peut écrire :

$$
E\left(y^{\mathrm{f}} y^{\mathrm{p} T}\right)=\Gamma_{\beta} E\left[x(k+\alpha) y^{\mathrm{p} T}\right]
$$

où l'expression $E(R)$ représente l'espérance mathématique de la matrice $R$.

Concrètement, on construit des matrices de Hankel « du passé $» Y^{\mathrm{p}}$ et du futur $Y^{\mathrm{f}}$ ainsi qu'une matrice des bruits $N^{\mathrm{f}}$ suivant la procédure générique $(\mathrm{P} 4)$.

L'équation (2) peut alors être étendue à l'ensemble des instants $k$ soit :

$$
Y^{\mathrm{f}}=\Gamma_{\beta} X_{\alpha}+N^{\mathrm{f}}
$$


où la matrice $X_{\alpha}$ est une bloc-ligne définie par la procédure (P5).

De l'équation (5) par multiplication à droite par $Y^{\mathrm{p} T}$ il vient :

$$
\Omega_{\beta}=\left(\frac{1}{N} Y^{\mathrm{f}} Y^{\mathrm{p} T}\right)_{N \rightarrow \infty}=\Gamma_{\beta}\left(\frac{1}{N} X_{\alpha} Y^{\mathrm{p} T}\right)_{N \rightarrow \infty}
$$

Une méthode un peu différente pour l'élimination du bruit a été proposée au début des années 90 par l'équipe Van Overshee-De Moor [9]. Dans cette méthode, le sous-espace des signaux « du futur » est projeté sur celui des signaux «du passé ». Les composantes des bruits «du futur » étant orthogonales à celles du passé, l'élimination asymptotique du bruit doit s'effectuer comme précédemment.

On comprendra aisément l'équivalence des deux méthodes si l'on examine l'expression de l'opérateur de projection : Les lignes de $Y^{\mathrm{f}}$ définissent un sous-espace de $R^{N}$, dont la projection sur le sous-espace ligne défini par la matrice $Y^{\mathrm{p}}$ est donnée par [9]

$$
\hat{O}_{\beta}=\frac{1}{N} Y^{\mathrm{f}} Y^{\mathrm{p} T}\left(Y^{\mathrm{p}} Y^{\mathrm{p} T}\right)^{\downarrow} Y^{\mathrm{p}}
$$

L'étape suivante consiste usuellement en une réduction d'espace. Si $l$ est le nombre de sorties observées du système, la décomposition en valeurs singulières (SVD) de la matrice $\hat{O}_{\beta}\left(\right.$ ou $\Omega_{\beta}$ ) fournit au plus $l \times \beta$ valeurs singulières non nulles, parmi lesquelles les $r$ plus grandes seulement seront retenues comme significatives

$$
\hat{\Omega}_{\beta}\left(\text { ou } \hat{O}_{\beta}\right)=\hat{Q} \hat{S} \hat{V}^{T}=\left[\hat{Q}_{\mathrm{s}} \tilde{Q}_{\mathrm{n}}\right]\left[\begin{array}{cc}
\hat{S}_{\mathrm{s}} & 0 \\
0 & \hat{S}_{\mathrm{n}}
\end{array}\right]\left[\begin{array}{c}
\hat{V}_{\mathrm{s}}^{T} \\
\hat{V}_{\mathrm{n}}^{T}
\end{array}\right]
$$

Les $r$ vecteurs singuliers gauches associés à ces valeurs singulières sont rassemblés dans la matrice $\hat{Q}_{\mathrm{s}}$. Dans cette équation, et dans la suite du texte, l'indication «^» indique que la variable affectée doit être considérée comme une estimation statistique de la même variable sans ce signe.

Par comparaison des équations (6) et (7), il est clair que l'on peut estimer $\Gamma_{\beta}$ à partir de $\hat{Q}_{\mathrm{s}}$. On choisit généralement, assez arbitrairement :

$$
\hat{\Gamma}_{\beta}=\hat{Q}_{\mathrm{s}} \hat{S}_{\mathrm{s}}^{1 / 2}
$$

De fait, cette matrice n'est définie qu'à une multiplication par une matrice arbitraire $T$ près. Il est facile de montrer que cette indétermination correspond à un simple changement de variables d'état dont la matrice $T$ est la matrice de passage.

À partir de la matrice $\hat{Q}_{\mathrm{s}}$ des estimations de la matrice de transition $A$ et de la matrice d'observation $C$ peuvent être obtenues en considérant la structure particulière de $\Gamma_{\beta}$, dite «structure shift », telle qu'illustrée par l'équation (4a). La référence [14] contient une analyse intéressante des propriétés particulières de ce type de matrice dans le domaine de l'identification des systèmes.

Une matrice $\bar{\Gamma}_{\beta}$ étant définie à partir de $\Gamma_{\beta}$ par suppression de la bloc-matrice supérieure, et de même une matrice $\underline{\Gamma}_{\beta}$ par suppression de la bloc-matrice inférieure on a évidemment la relation

$$
\bar{\Gamma}_{\beta}=\underline{\Gamma}_{\beta} A
$$

On posera :

$$
\underline{\Gamma}_{\beta}=J_{1} \hat{\Gamma}_{\beta} \quad \text { avec } \quad J_{1}=\left[I_{l(\alpha-1)} 0_{l(\alpha-1), l}\right]
$$

et

$$
\bar{\Gamma}_{\beta}=J_{2} \hat{\Gamma}_{\beta} \quad \text { avec } \quad J_{2}=\left[0_{l(\alpha-1), l} I_{l(\alpha-1)}\right]
$$

L'équation (9) peut être résolue par moindres carrés soit

$$
\hat{A}=\left(J_{1} \hat{\Gamma}_{\beta}\right)^{\downarrow} J_{2} \hat{\Gamma}_{\beta}
$$

où le symbole « $\downarrow$ désigne la pseudo-inverse d'une matrice.

Une estimation de la matrice $C$ peut être prélevée directement dans la matrice $\Gamma_{\beta}$ comme l'indique l'équation (4a).

De manière classique, les valeurs propres de la matrice $A$ fournissent les pulsations propres et amortissements modaux, et les vecteurs propres les déformées modales. De fait, la matrice $A$ n'est obtenue qu'à une similitude près, moyennant un changement de base de matrice $T$ inconnue. La matrice $C$ est également définie au facteur matriciel $T$ près. Mais ici à nouveau cette indétermination est sans conséquence puisqu'elle ne change pas les valeurs propres de $A$, et que les vecteurs propres peuvent être ramenés dans l'espace physique des sorties par la matrice $C$.

Bien qu'équivalente, l'approche Van OversheeDe Moor a sur l'approche N4SID un avantage important, à savoir qu'elle donne un sens au terme conjoint de $\Gamma_{\beta}$ dans la SVD. Ainsi est démontrée la relation suivante [9] :

$$
\hat{O}_{\beta}=\hat{\Gamma}_{\beta} \hat{X}_{\alpha}
$$

dans laquelle la matrice $\hat{X}_{\alpha}$ est une matrice bloc-ligne des états prévisionnels résultant des filtres de Kalman constitués, pour chaque colonne de la matrice $Y^{\mathrm{p}}$, à partir des données de cette colonne [9].

Van Overshee [9] démontre qu'une estimation non biaisée des matrices $A$ et $C$ peut être obtenue par résolution inverse (moindres carrés) de l'équation

$$
\left[\begin{array}{c}
\hat{X}_{\alpha+1} \\
Y_{\alpha}
\end{array}\right]=\left[\begin{array}{c}
A \\
C
\end{array}\right] \hat{X}_{\alpha}
$$

où $Y_{\alpha}$ est la matrice formée d'une ligne de $N$ vecteurs de sortie à partir de l'indice $\alpha$ (procédure (P5)).

Cette même équation permet l'évaluation des « bruits du système ». On démontre [9] qu'une estimation non biaisée de ces bruits est donnée par :

$$
\mid \begin{aligned}
& \hat{\rho}_{w}=\hat{X}_{\alpha+1}-\hat{A} \hat{X}_{\alpha} \\
& \hat{\rho}_{v}=Y_{\alpha}-\hat{C} \hat{X}_{\alpha}
\end{aligned}
$$

Ce résultat sera important lors de l'analyse statistique des résultats d'identification. 


\section{L'analyse statistique des paramètres modaux}

Les bases de la théorie qui suit ont été établies par plusieurs auteurs, dans un cadre légèrement différent [15-17]. Dans ces articles, le contexte choisi est « déterministestochastique », et le développement est centré sur les approches de type 4SID classiques ou approchantes. Les lignes qui suivent reprennent la méthode en l'adaptant au contexte purement stochastique, cependant dans le cadre de l'approche Van Overshee-De Moor [9]. La méthode comporte plusieurs étapes : i) calcul de l'erreur d'estimation des paramètres modaux (méthode du shift), ii) démonstration de la normalité asymptotique de ces paramètres, iii) calcul des covariances des paramètres modaux.

\subsection{Erreur d'estimation des paramètres modaux}

Supposons la matrice de transition $A$ identifiée par la méthode du « shift » (cf. Sect. 3.2), $T$ la matrice qui diagonalise la matrice $A$. L'estimée de la matrice $\Gamma_{\beta}$ par la méthode d'identification sera notée $\hat{\Gamma}_{\beta}$ et celle de $A$ sera notée $\hat{A}$. L'erreur $\tilde{A}$ commise sur la matrice $A$ est, compte tenu de l'équation (9) :

$$
\tilde{A}=\hat{A}-A=\left(J_{1} \hat{\Gamma}_{\beta}\right)^{\downarrow}\left(J_{2} \hat{\Gamma}_{\beta}-J_{1} \hat{\Gamma}_{\beta} A\right)
$$

À ce stade, il y a lieu de faire deux approximations. Il est clair que l'erreur $\tilde{A}$, au premier ordre, provient du second facteur de ce produit. Au premier ordre on peut donc remplacer dans le premier facteur l'estimé $\hat{\Gamma}_{\beta}$ par sa valeur exacte $\Gamma_{\beta}$. Par ailleurs, la matrice des valeurs propres étant

$$
A_{\mathrm{d}}=\operatorname{diag}\left(\mu_{k}\right)=T A T^{-1}
$$

on démontre que l'erreur sur ces valeurs propres peut être approximée, au premier ordre, par l'équation :

$$
\tilde{\mu}_{k}=T_{k} \cdot \tilde{A} T_{. k}^{-1}
$$

Dans cette expression $T_{k}$. représente la ligne $k$ de la matrice $T$ et $T_{. k}^{-1}$ la colonne $k$ de la matrice $T^{-1}$.

Des équations (11) et (13) et compte tenu des précédentes remarques on peut écrire :

$$
\tilde{\mu}_{k} \approx T_{k .}\left(J_{1} \Gamma_{\beta}\right)^{\downarrow}\left(J_{2}-J_{1} \mu_{k}\right) \hat{\Gamma}_{\beta} T_{. k}^{-1} .
$$

Compte tenu de l'équation (8) et en posant

$$
f_{k}^{*}=T_{k .}\left(J_{1} \Gamma_{\beta}\right)^{\downarrow}\left(J_{2}-J_{1} \mu_{k}\right)
$$

cette expression se réduit à :

$$
\tilde{\mu}_{k} \approx f_{k}^{*} \hat{Q}_{\mathrm{s}} S_{\mathrm{s}}^{1 / 2} T_{. k}^{-1}
$$

Dans ces expressions, l'indice supérieur «* $*$ indique la «transposée conjuguée » de la matrice.

\subsection{Normalité asymptotique de l'erreur sur les paramètres modaux}

La démonstration ci-après reprend celle proposée dans la référence [17] et la prolonge dans le cadre de l'approche Van Overshee-De Moor. La SVD définie par l'équation (7) permet d'écrire :

$$
\hat{O}_{\beta}=\frac{1}{N} Y^{\mathrm{f}} Y^{\mathrm{p} T}\left(Y^{\mathrm{p}} Y^{\mathrm{p} T}\right)^{\downarrow} Y^{\mathrm{p}}=\hat{Q}_{\mathrm{s}} \hat{S}_{\mathrm{s}} \hat{V}_{\mathrm{s}}^{T}+\hat{Q}_{\mathrm{n}} \hat{S}_{\mathrm{n}} \hat{V}_{\mathrm{n}}^{T}
$$

Multiplions cette équation à droite par $\hat{V}_{\mathrm{s}} \hat{S}_{\mathrm{s}}^{-1}$. Compte tenu de l'orthogonalité des colonnes de $\hat{V}_{\mathrm{n}}$ et $\hat{V}_{\mathrm{s}}$, il vient :

$$
\hat{Q}_{S}=\frac{1}{N} Y^{\mathrm{f}} Y^{\mathrm{p} T}\left(Y^{\mathrm{p}} Y^{\mathrm{p} T}\right)^{\downarrow} Y^{\mathrm{p}} \hat{V}_{\mathrm{s}} \hat{S}_{\mathrm{s}}^{-1}
$$

soit, dans l'équation (15)

$$
\tilde{\mu}_{k} \approx \frac{1}{N} f_{k}^{*} Y^{\mathrm{f}} Y^{\mathrm{p} T}\left(Y^{\mathrm{p}} Y^{\mathrm{p} T}\right)^{\downarrow} Y^{\mathrm{p}} \hat{V}_{\mathrm{s}} \hat{S}_{\mathrm{s}}^{-1 / 2} T_{. k}^{-1} .
$$

En pratique, il est intéressant de transformer l'expression de la matrice $\hat{V}_{\mathrm{s}}$, dont la taille $(N, N-r)$ peut être considérable. Multipliant à gauche l'équation (16) par $\hat{S}_{\mathrm{s}}^{-1} \hat{Q}_{\mathrm{S}}^{T}$ il vient :

$$
\hat{V}_{\mathrm{s}}=\hat{O}_{\beta}^{T} \hat{Q}_{\mathrm{s}} \hat{S}_{\mathrm{s}}^{-1}
$$

d'où l'on déduit

$$
\tilde{\mu}_{k} \approx f_{k}^{*}\left(\frac{1}{N} Y^{\mathrm{f}} Y^{\mathrm{p} T}\right) \hat{l}_{k}
$$

en posant

$$
\hat{l}_{k}=\left(Y^{\mathrm{p}} Y^{\mathrm{p} T}\right)^{\downarrow} Y^{\mathrm{p}} Y^{\mathrm{f} T} \hat{S}_{\mathrm{s}}^{-3 / 2} T_{. k}^{-1} .
$$

Compte tenu de l'équation (5), il vient :

$$
\tilde{\mu}_{k} \approx f_{k}^{*}\left(\frac{1}{N} \Gamma_{\beta} X_{i} Y^{\mathrm{p} T}\right) \hat{l}_{k}+f_{k}^{*}\left(\frac{1}{N} N^{\mathrm{f}} Y^{\mathrm{p} T}\right) \hat{l}_{k}
$$

Le premier terme s'élimine en vertu de la propriété [16] :

$$
f_{k}^{*} \Gamma_{\beta}=0
$$

Démonstration : De l'équation (14), on déduit :

$$
f_{k}^{*} \Gamma_{\beta}=T_{k .}\left(J_{1} \Gamma_{\beta}\right)^{\downarrow}\left(J_{2}-J_{1} \mu_{k}\right) \Gamma_{\beta}
$$

soit

$$
\begin{aligned}
& f_{k}^{*} \Gamma_{\beta}=T_{k .}\left(J_{1} \Gamma_{\beta}\right)^{\downarrow}\left(J_{1} \Gamma_{\beta} A-J_{1} \mu_{k}\right) \Gamma_{\beta}= \\
& T_{k .}\left(J_{1} \Gamma_{\beta}\right)^{\downarrow}\left(J_{1} \Gamma_{\beta}\right)\left(A-\mu_{k} I\right)=T_{k .}\left(A-\mu_{k} I\right)
\end{aligned}
$$

Compte de l'équation (12), on a : $T A=A_{\mathrm{d}} T$ dont la

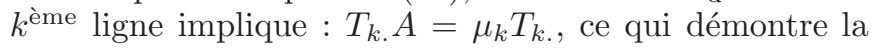
relation (18).

Au second terme de l'équation (17), $\hat{l}_{k}$ tend asymptotiquement vers un vecteur déterministe :

$$
\hat{l}_{k} \rightarrow l_{k}=\left[R_{y_{. \alpha} y_{. \alpha}}(0)\right]^{\downarrow} R_{y_{. \alpha} y_{. \beta}}(\alpha) Q_{\mathrm{s}} S_{\mathrm{s}}^{-3 / 2} T_{. k}^{-1}
$$


où une matrice $R_{u v}(\tau)$ représentent la matrice de covariance d'un vecteur $u$ avec un vecteur $v$ pour le décalage $\tau$ :

$$
R_{u v}(\tau)=E\left(u(t+\tau) v^{T}(t)\right)
$$

L'équation (19) devient, pour $N$ infini

$$
\tilde{\mu}_{k} \approx \frac{1}{\sqrt{N}} f_{k}^{*}\left(\frac{1}{\sqrt{N}} N^{\mathrm{f}} Y^{\mathrm{p} T}\right) l_{k}
$$

Dans ce produit, le terme central représente la somme d'un grand nombre de termes aléatoires indépendants. Le théorème central limite démontre la normalité asymptotique de l'erreur sur $\mu_{k}$.

Le taux d'amortissement $\eta_{k}$ se déduit de $\mu_{k}$ par la relation

$$
\mu_{k}=\mathrm{e}^{\lambda_{k} T}
$$

où l'on a :

$$
\lambda_{k}=-\omega_{k} \eta_{k} \pm j \omega_{k} \sqrt{1-\eta_{k}^{2}}
$$

et où $\omega_{k}$ est la pulsation modale correspondant à la valeur propre $\mu_{k}$. On a alors :

$$
\eta_{k}=-\frac{\lambda_{k}+\lambda_{k}^{(\mathrm{c})}}{2 \omega_{k}}
$$

où l'indice supérieur (c) indique le terme conjugué.

On sait que la pulsation modale $\omega_{k}$, qui est une valeur stationnaire du quotient de Rayleigh est obtenue avec une précision du second ordre. Par ailleurs, de l'équation (21), on tire, au premier ordre :

$$
\tilde{\mu}_{k}=\tilde{\lambda}_{k} T \mu_{k}
$$

On en déduit l'erreur sur l'amortissement :

$$
\tilde{\eta}_{k} \approx-\frac{1}{2 \omega_{k} T}\left(\frac{\tilde{\mu}_{k}}{\mu_{k}}+\frac{\tilde{\mu}_{k}^{(\mathrm{c})}}{\mu_{k}^{(\mathrm{c})}}\right)
$$

soit, de l'équation (20) :

$$
\begin{aligned}
\tilde{\eta}_{k}=\frac{-1}{2 \sqrt{N} \omega_{k} T}\left[f _ { k } ^ { * } \left(\frac{1}{\sqrt{N}}\right.\right. & \left.N^{\mathrm{f}} Y^{\mathrm{p} T}\right) \frac{l_{k}}{\mu_{k}} \\
& \left.+\frac{l_{k}^{*}}{\mu_{k}^{*}}\left(\frac{1}{\sqrt{N}} N^{\mathrm{f}} Y^{\mathrm{p} T}\right) f_{k}\right]
\end{aligned}
$$

que l'on peut expliciter en introduisant les composantes $f_{k, i}, l_{k, j}$ des vecteurs $f_{k}$ et $l_{k}$ :

$$
\begin{aligned}
\tilde{\eta}_{k}= & \frac{-1}{2 \sqrt{N} \omega_{k} T} \sum_{i=1, \alpha} \sum_{j=1, \beta} \\
& \times\left[\left(\frac{f_{k, j}^{(\mathrm{c})} l_{k, i}}{\mu_{k}}+\frac{l_{k, j}^{(\mathrm{c})} f_{k, i}}{\mu_{k}^{(\mathrm{c})}}\right) \frac{1}{\sqrt{N}} \sum_{l=1, N} N_{j l}^{\mathrm{f}} Y_{i l}^{\mathrm{p}}\right]
\end{aligned}
$$

Le dernier terme de cette équation apparaît comme la somme d'un grand nombre de variables aléatoires indépendantes de moyenne nulle. Le théorème central limite nous indique que la distribution de cette somme est asymptotique à une variable normale de moyenne nulle.

\subsection{Covariance des erreurs d'amortissement}

Les covariances des erreurs d'amortissement se déduisent de la formule suivante :

$$
\begin{aligned}
\operatorname{cov}\left(\tilde{\eta}_{i}, \tilde{\eta}_{j}\right) & =E\left(\tilde{\eta}_{i} \tilde{\eta}_{j}\right) \\
& =\frac{1}{4 \omega_{i} \omega_{j} T^{2}} E\left(\frac{\tilde{\mu}_{i} \tilde{\mu}_{j}}{\mu_{i} \mu_{j}}+\frac{\tilde{\mu}_{i} \tilde{\mu}_{j}^{*}}{\mu_{i} \mu_{j}^{*}}+\frac{\tilde{\mu}_{i}^{*} \tilde{\mu}_{j}}{\mu_{i}^{*} \mu_{j}}+\frac{\tilde{\mu}_{i}^{*} \tilde{\mu}_{j}^{*}}{\mu_{i}^{*} \mu_{j}^{*}}\right)
\end{aligned}
$$

Le calcul des 4 termes s'effectue rapidement en utilisant la formule ci-après : $\xi, \xi^{\prime}, \lambda, \lambda^{\prime}$ étant 4 vecteurs de dimensions respectives $l \beta, l \beta, l \alpha, l \alpha$, les scalaires

$$
\rho=\xi *\left(\frac{1}{\sqrt{N}} N^{\mathrm{f}} Y^{\mathrm{p} T}\right) \lambda
$$

et

$$
\rho^{\prime}=\xi^{\prime} *\left(\frac{1}{\sqrt{N}} N^{\mathrm{f}} Y^{\mathrm{p} T}\right) \lambda^{\prime}
$$

ont pour covariance :

$$
\operatorname{cov}\left(\rho, \rho^{\prime}\right)=\sum_{|\tau|<\alpha} \lambda^{T} R_{y_{. \alpha} y_{. \alpha}}(\tau) \lambda^{\prime} \xi^{\prime} * R_{n_{. \beta} n_{. \beta}}(\tau) \xi^{(\mathrm{c})}
$$

Cette formule est démontrée succinctement dans la référence [16], et peut être vérifiée par un examen minutieux des produits figurant dans l'expression et de la dépense stochastique des termes qui les composent.

En appliquant cette équation aux quatre termes de l'équation (27), et en regroupant les termes conjugués, il vient :

$$
\begin{aligned}
& \operatorname{cov}\left(\tilde{\eta}_{i}, \tilde{\eta}_{j}\right)=\frac{1}{\omega_{i} \omega_{j} T^{2}} \sum_{|\tau|<\alpha} \\
& \times \operatorname{Re}\left[\frac{1}{\mu_{i}} l_{i}^{T} R_{y_{. \alpha} y_{. \alpha}}(\tau) \operatorname{Re}\left(\frac{l_{j} f_{j}^{*}}{\mu_{j}}\right) R_{n_{. \beta} n_{. \beta}}(\tau) f_{i}^{(\mathrm{c})}\right]
\end{aligned}
$$

Noter que la matrice $R_{n_{\beta} n_{\beta} \beta}(\tau)$ peut être construite, compte tenu de l'équation (3), si l'on dispose d'une évaluation des bruits de système $w$ et $v$. Rappelons que cette évaluation a été obtenue par l'équation (10).

\section{Validation de la méthode}

\subsection{Simulations numériques}

Un système à $n$ degrés de liberté est modélisé à l'aide de $n$ oscillateurs masse-amortisseur-ressort indépendants (Fig. 1). Chaque sous-système est excité indépendamment par un bruit blanc gaussien $w^{(k)}$. Un couplage des sorties est réalisé à volonté en combinant les $n$ signaux de réponse dans $l$ signaux composés, à travers une matrice d'observation $C$ arbitraire. Un bruit de mesure blanc gaussien indépendant $v(t)$ est ajouté sur les sorties. 
Tableau 1. Exemple à 2 ddl.

\begin{tabular}{|c|c|c|c|c|c|c|c|}
\hline & & \multicolumn{2}{|c|}{ Mode 1} & \multicolumn{4}{|c|}{ Mode 2} \\
\hline Puls & $: \omega_{k} T$ & \multicolumn{2}{|c|}{1 , } & \multicolumn{2}{|c|}{2,} & & \\
\hline Amortiss & nt $\eta_{k} \times 100$ & \multicolumn{2}{|c|}{2 , } & \multicolumn{2}{|c|}{1,} & & \\
\hline \multicolumn{2}{|c|}{ Bruit de process : $\sigma$} & 0,5 & 0 & 1 , & 0 , & & \\
\hline Sortie 1 & Matrice & 1 & 0 & 0,5 & 0 & Bruit de & 0,25 \\
\hline Sortie 2 & $\mathrm{C}$ & 0 & 0 & 0 & 1, & mesure $: \sigma$ & 0,25 \\
\hline \multicolumn{2}{|c|}{ Moy $\left(\omega_{k} T\right)$} & \multicolumn{2}{|c|}{1,0001} & \multicolumn{2}{|c|}{2,0001} & \multicolumn{2}{|c|}{ Statistique } \\
\hline & $T)$ & \multicolumn{2}{|c|}{0,22} & \multicolumn{2}{|c|}{0,22} & \multicolumn{2}{|c|}{200 échantillons } \\
\hline Moy & $\times 100)$ & \multicolumn{2}{|c|}{2,03} & \multicolumn{2}{|c|}{1,01} & \multicolumn{2}{|l|}{ de } \\
\hline & 100) & \multicolumn{2}{|c|}{0,25} & \multicolumn{2}{|c|}{0,10} & \multicolumn{2}{|c|}{5000 points } \\
\hline \multicolumn{6}{|c|}{ Écart-types amortissements prévisionnels } & \multicolumn{2}{|c|}{ Échantillon } \\
\hline \multirow{5}{*}{\multicolumn{2}{|c|}{$\sigma\left(\eta_{k}\right) \times 100$ prévisionnel }} & \multicolumn{2}{|c|}{0,31} & \multicolumn{2}{|c|}{0,12} & \multicolumn{2}{|l|}{1} \\
\hline & & & & \multicolumn{2}{|c|}{0,11} & \multicolumn{2}{|l|}{2} \\
\hline & & \multicolumn{2}{|c|}{0,32} & \multicolumn{2}{|c|}{0,12} & \multicolumn{2}{|l|}{3} \\
\hline & & \multicolumn{2}{|c|}{0,31} & \multicolumn{2}{|c|}{0,11} & \multicolumn{2}{|l|}{4} \\
\hline & & \multicolumn{2}{|c|}{0,31} & \multicolumn{2}{|c|}{0,12} & \multicolumn{2}{|l|}{5} \\
\hline
\end{tabular}

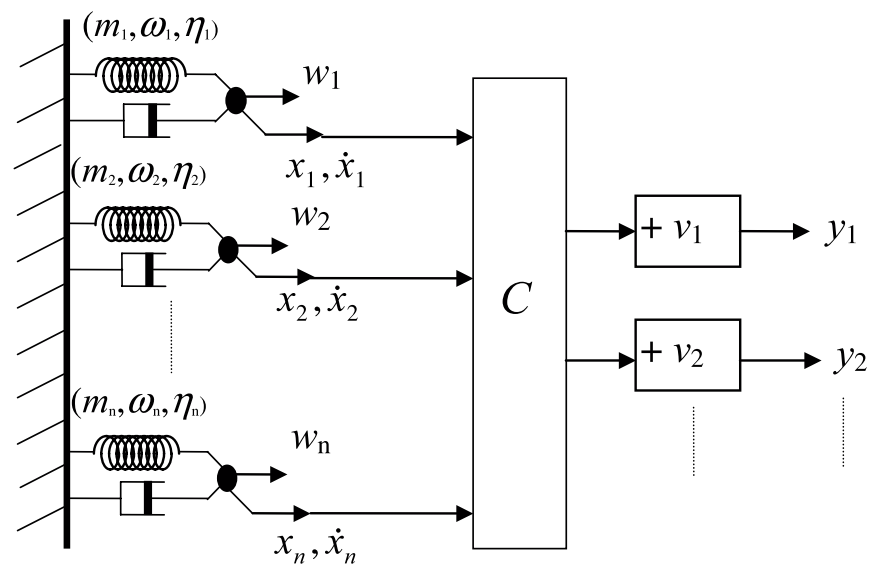

Fig. 1. Simulation d'un système à $n$ degrés de liberté.

\section{a) Exemple à 2 degrés de liberté}

Les données du système figurent dans le tableau 1. Le diagramme de stabilisation a montré que les meilleurs paramètres d'identification sont $\alpha=\beta=r=4$.

Le tableau 1 montre les résultats d'une statistique brute d'identification, réalisée à partir de 200 échantillons de taille 5000 des sorties du système. La « prévision statistique » de l'erreur d'identification, conforme à la théorie ci-dessus, est réalisée indépendamment sur les 5 premiers échantillons (taille 5000). On constate que la prévision statistique de l'erreur d'identification des amortissements est toujours très proche, pour un échantillon unique de la dispersion statistique obtenue avec les 200 échantillons, et qu'elle varie peu suivant l'échantillon.

En fonction du nombre de données $N$, les résultats démontrent une évolution très précise en $1 / \sqrt{N}$. L'intérêt de cette constatation est qu'elle permet d'anticiper la taille de l'échantillon nécessaire si l'on veut assurer une précision donnée, au sens statistique (par exemple une précision choisie de $\pm 2 \sigma$ pour $95 \%$ de confiance).

\section{b) Exemple à 5 degrés de liberté}

Le système est formé de 5 éléments de même type que précédemment. Les caractéristiques des sous-systèmes sont données en-tête du tableau 2. La matrice $C$ qui effectue le couplage des sorties indépendantes, les niveaux de bruit sur chaque canal et sur les sorties sont également indiqués dans le tableau.

Une identification avec évaluation prévisionnelle de l'erreur sur les amortissements est effectuée sur un échantillon de 5000 valeurs. Les résultats donnent des écarts-types prévisionnels sur les erreurs d'amortissement importants, de l'ordre de grandeur ou supérieurs aux valeurs mêmes des amortissements. Un passage à 50000 valeurs devrait diviser ces fourchettes par $\sqrt{10} \approx 3,2$ environ. Les résultats de deux passages avec $N=50000$ sont indiqués sur le même tableau.

L'analyse des résultats montre que la règle d'évolution de l'écart-type prévisionnel en $1 / \sqrt{N}$ reste vérifiée avec précision. Ces écart-types prévisionnels paraissent corrects pour certaines fréquences, cependant trop grands pour les fréquences 3 et surtout 4 , au regard de la bonne qualité des résultats réels. De fait, l'imprécision semble concerner autant les pulsations que les amortissements. On peut en conclure que la qualité de l'écart-type prévisionnel est très affectée par la qualité générale de l'identification pour chaque mode.

\subsection{Exemple expérimental}

Des données expérimentales sont recueillies en soumettant en laboratoire un système comportant 3 poutres et deux masses à l'action d'un pot vibrant. L'excitation est un bruit blanc dont les valeurs sont ignorées. 
Tableau 2. Exemple à 5 ddl.

\begin{tabular}{|c|c|c|c|c|c|c|c|c|c|c|c|c|}
\hline Mode & \multicolumn{2}{|c|}{ Mode 1} & \multicolumn{2}{|c|}{ Mode 2} & \multicolumn{2}{|c|}{ Mode 3} & \multicolumn{2}{|c|}{ Mode 4} & \multicolumn{4}{|c|}{ Mode 5} \\
\hline Pulsation : $\omega_{k} T$ & \multicolumn{2}{|c|}{0,5} & \multicolumn{2}{|c|}{1,} & \multicolumn{2}{|c|}{1,5} & \multicolumn{2}{|c|}{1,8} & \multicolumn{2}{|c|}{2 , } & & \\
\hline Amortissement $\eta_{k} \times 100$ & \multicolumn{2}{|c|}{2} & \multicolumn{2}{|c|}{1 , } & \multicolumn{2}{|c|}{3 , } & \multicolumn{2}{|c|}{2 , } & \multicolumn{2}{|c|}{1 , } & & \\
\hline Bruit de process : $\sigma$ & 1, & 0 & 0,5 & 0 , & 0,3 & 0 & 0,7 & 0 , & 0,5 & 0 & & \\
\hline Sortie 1 & 0,5 & 0 & 1 & 0 & 0,7 & 0 & 0,4 & 0 & 0,6 & 0 & Bruit de & 0,25 \\
\hline Sortie 2 & 0,2 & 0 & 0,5 & 0 & 0,3 & 0 & 0,7 & 0 & 0,4 & 0 & mesure $: \sigma$ & 0,2 \\
\hline$\omega_{k} T$ & \multicolumn{2}{|c|}{0,4993} & \multicolumn{2}{|c|}{0,9997} & \multicolumn{2}{|c|}{1,5770} & \multicolumn{2}{|c|}{1,7962} & \multicolumn{2}{|c|}{1,9969} & \multicolumn{2}{|c|}{ Identification 1} \\
\hline$\eta_{k} \times 100$ & \multicolumn{2}{|c|}{1,82} & \multicolumn{2}{|c|}{0,9} & \multicolumn{2}{|c|}{3,64} & \multicolumn{2}{|c|}{0,73} & \multicolumn{2}{|c|}{1,01} & \multicolumn{2}{|c|}{ Échantillon } \\
\hline$\sigma\left(\eta_{k}\right) \times 100$ prévisionnel & \multicolumn{2}{|c|}{1,92} & \multicolumn{2}{|c|}{0,63} & \multicolumn{2}{|c|}{3,57} & \multicolumn{2}{|c|}{5,62} & \multicolumn{2}{|c|}{0,54} & \multicolumn{2}{|c|}{$N=5000$} \\
\hline$\omega_{k} T$ & \multicolumn{2}{|c|}{0,5} & 0,9 & & 1,56 & & $1,7 \mathrm{~s}$ & & 2,00 & & Identificat & n 2 \\
\hline$\eta_{k} \times 100$ & 2,0 & & 1 & & 2,3 & & 2,1 & & 1,0 & & Échantil & \\
\hline$\sigma\left(\eta_{k}\right) \times 100$ prévisionnel & 0,6 & & 0 & & 1,3 & & 1,5 & & 0,1 & & $N=50$ & \\
\hline$\omega_{k} T$ & 0,50 & & $\overline{0,9}$ & & 1,56 & & $\overline{1,80}$ & & 2,00 & & Identificat & n 3 \\
\hline$\eta_{k} \times 100$ & 2,0 & & 0 & & 3,2 & & 1, & & $0, s$ & & Échantil & \\
\hline$\sigma\left(\eta_{k}\right) \times 100$ prévisionnel & 0,6 & & 0 & & 1,2 & & 1,7 & & 0,1 & & $N=50$ & \\
\hline
\end{tabular}

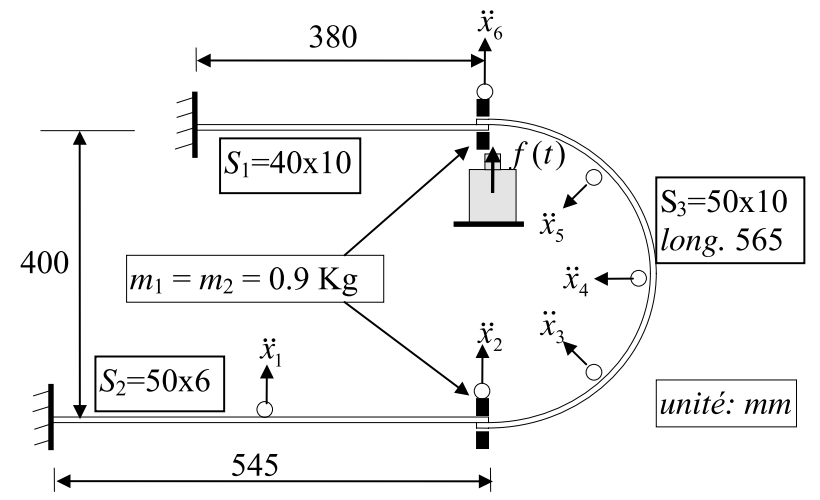

Fig. 2. Dispositif expérimental.

Les réponses du système sont recueillies sur 6 voies accélérométriques (Fig. 2).

L'observation des spectres des sorties montre que cette structure possède deux modes fortement dominants. Une étude de stabilisation confirme cette hypothèse avec la possibilité d'un troisième mode mal défini. De cette étude on conclut que les meilleurs ordres d'identification sont $\alpha=\beta=r=6$.

Une étude statistique est menée sur la base de 20 échantillons de 2000 valeurs. Indépendamment une évaluation de l'écart-type prévisionnel des amortissements est effectuée sur le premier de ces échantillons. Pour les deux modes « certains », les écarts-types prévisionnels sont en concordance raisonnables avec les résultats statistiques. Pour le troisième mode « incertain », dont l'amortissement identifié est très élevé (22 \%), l'écart-type prévisionnel est environ 4 fois trop faible. Ce résultat confirme les conclusions de la simulation précédente.

\section{Conclusions}

Les développements ci-dessus ont montré que, dans le principe, la procédure d'identification par sous-espaces était capable de fournir, en même temps que des estimations des paramètres, des écart-types «prévisionnels » pour ces valeurs sans qu'il soit nécessaire de pratiquer une coûteuse statistique de résultats.

Les simulations confirment cette possibilité. Toutefois les simulations à grand nombre de modes donnent des résultats inégaux. Il est vrai que pour certains modes les écart-types «prévisionnels» sont obtenus avec une très forte surestimation. Si ce résultat va dans un sens plutôt favorable, il peut amener à invalider à tort des résultats pourtant relativement précis.

L'étude sur un cas réel amène sensiblement les mêmes conclusions, quoique le sur-encadrement des valeurs estimées ne soit plus systématique.

La raison de cette imprécision est facile à comprendre lorsqu'on examine le développement théorique. L'évaluation de l'erreur d'estimation repose sur l'évaluation d'une différence entre deux expressions dont certains termes comportent des valeurs déterministes inconnues qu'il faut bien remplacer par des estimations. Malgré cela l'estimation prévisionnelle d'erreur apparaît robuste et généralement fiable.

Les perspectives futures devront s'orienter vers un affinement de l'analyse afin de resserrer plus précisément les fourchettes de validation des paramètres.

À l'issue de ces travaux d'intéressantes applications se profilent, tels le contrôle en temps réel de certaines structures, en particulier d'avions en vol dans des situations proches du «flottement ». D'une manière plus générale, il est probable que l'analyse modale des structures « en service » devrait se substituer de plus en plus fréquemment à l'analyse modale «sur banc», en ce sens qu'elle fournit des informations plus réalistes. Dans ce cas les informations de type statistique prendront une importance majeure. Cet article est une contribution dans ce sens. 
Tableau 3. Exemple expérimental.

\begin{tabular}{|c|c|c|c|c|}
\hline Mode & Mode 1 & Mode 2 & Mode 3 & \\
\hline \multicolumn{5}{|c|}{ Analyse statistique : 20 échantillons de 2000 points } \\
\hline $\operatorname{Moy}\left(\omega_{k} T\right)$ & 0,7316 & 2,0141 & 2,4988 & \\
\hline$\sigma\left(\omega_{k} T\right)$ & 0,0011 & 0,0019 & 0,0557 & \\
\hline $\operatorname{Moy}\left(\eta_{k} \times 100\right)$ & 0,64 & 0,35 & 22,5 & \\
\hline$\sigma\left(\eta_{k} \times 100\right)$ & 0,21 & 0,10 & 2,5 & \\
\hline \multicolumn{4}{|c|}{ Écart-types prévisionnels des amortissements } & Échantillon \\
\hline \multirow{8}{*}{$\sigma\left(\eta_{k}\right) \times 100$} & 2,8 & 0,68 & 5,4 & 1. $N=100$ \\
\hline & 1,8 & 0,29 & 4,9 & 2. $N=200$ \\
\hline & 1,23 & 0,12 & 3,5 & 3. $N=500$ \\
\hline & 0,6 & 0,1 & 2,2 & 4. $N=1000$ \\
\hline & 0,42 & 0,07 & 1,7 & 5. $N=2000$ \\
\hline & 0,28 & 0,05 & 1,3 & 6. $N=4000$ \\
\hline & 0,1 & 0,02 & 0,56 & 7. $N=24000$ \\
\hline & 0,08 & 0,02 & 0,44 & 8. $N=40000$ \\
\hline
\end{tabular}

\section{Références}

[1] D.J. Ewins, Modal testing: theory and practice, John Wiley \& Sons, New York, 1985

[2] L. Ljung, System Identification, Theory for the User, Prentice Hall, Englewood Cliffs, NJ, 1987

[3] S.M. Kay, Modal spectral estimation : theory and application, Prentice Hall, Englewood Cliffs, NJ, 1988

[4] J. Leuridan, D.L. Brown, R.J. Allemang, Time domain parameter identification for linear modal analysis: a unifying approach, J. Vibrations, Acoustics, Stress and Reliability in design 108 (1986) 1-7

[5] F. Deblauwe, D.L. Brown, R.J. Allemang, The polyreference time domain technique, Proc. 5th IMAC, 1987, pp. $832-845$

[6] J.N. Juang, Applied System Identification, Prentice Hall Englewood Cliffs, NJ, 1994

[7] M. Viberg, Subspace-based methods for the identification of linear time-invariant systems, Automatica 31(12) (1995) 1835-1851

[8] P. Van Overshee, B. De Moor, N4SID: Subspace Algorithms for the Identification of Combined Deterministic Stochastic Systems, Automatica 30 (1994) 75-93

[9] O. Van Overshee, B. De Moor, Subspace Identification for Linear Systems, Dordrecht: Kluwer Academic Publications, 1996

[10] M. Verhaegen, P. Dewilde, Subspace Model Identification. Part I: The Output-error State Space Model Identification Class of Algorithms, Int. J. Control 56(5) (1992) 1187-1210
[11] M. Abdelghani, M. Verhaegen, P. Van Overshee, B. De Moor, Comparison study of subspace identification methods applied to flexible structures, Mechanical Systems and Signal Processing 12(5) (1998) 679-692

[12] P. Van Overshee, B. De Moor, A unifying theorem of three subspace identification algorithms, Automatica 31(12) (1995) 1853-1864

[13] T. Kailath, Linear Systems, Prentice Hall, Englewood Cliffs, NJ, 1980

[14] B. De Moor, Mathematical Concepts and Techniques for Modeling of Static and Dynamic Systems, Doctoral Dissertation, Department of Electrical Engineering, Kath. Universiteit Leuven, Belgium, 1988

[15] M. Viberg, B. Ottersen, B. Wahlberg, L. Ljung, A statistical perspective on state space modeling using subspace methods, Proc. 30th Int. Conf. on Decision and Control, Brighton UK, 1991, pp. 1337-1342

[16] M. Jansson, B. Wahlberg, A linear regression approach to state-space subspace system identification, Signal Processing 52 (1996) pp. 103-129

[17] M. Viberg, B. Wahlberg, B. Ottersen, Analysis of statespace system identification methods based on instrumental variables and subspace fitting, Automatica 33(9) (1997) 1603-1616

[18] P.E. Gautier, C. Gontier, M. Smail, Robustness of a ARMA identification method for modal analysis of mechanical systems in the presence of noise, J. Sounds and Vibration 179(2) (1995) 227-242 